\title{
Simulation of wind power plant with double-fed machine when operating in autonomous system in parallel with synchronous commensurable power generator
}

\author{
Rauf Mustafayev ${ }^{1}$, Laman Hasanova ${ }^{1, *}$ \\ ${ }^{1}$ Azerbaijan Scientific-Research and Designed-Prospecting Institute of Energetics, pr. Zardabi 94 , Azerbaijan
}

\begin{abstract}
Autonomous system, containing synchronous generators in a classic design, driven by either diesel or gas turbine, which operates in parallel with a wind power plant (WPP) containing an asynchronous double-fed machine (ADFM) through external network, is considered in the paper. At the same time, the power transmission scheme and the corresponding equivalent circuit are also drawn up. The equations of the synchronous generator and the external network are written in the axes rotating at the speed of the synchronous generator rotor, and the equations of the WPP's generator in the axes rotating at the speed of its rotor. By means of "docking" equations a digital model of the entire system is compiled with the external network parameters and loads on the side of the synchronous generator and WPP's asynchronous generator, which are represented by constant-conductivity shunts. Static and dynamic characteristics of the system are calculated.
\end{abstract}

\section{Introduction}

Autonomous commensurable power systems of are widely used in various sectors of national economy. When using wind power plants in the autonomous power supply system, naturally in places with high wind power potential, the problem of studying the conditions of parallel operation arises, when the output power of wind power plants changes depending on the wind speed value. Such systems can be used in regions without centralized power supply - mountainous villages, oil production platforms ("Oil rocks" type), etc.

\section{Materials and methods}

The electricity transmission diagram is presented in Fig. 1. Here synchronous generator SG, generator voltage of which is about $10.5 \mathrm{kV}$, feeds the load 1 from generator buses. Further, the step-up transformer $\mathrm{T}_{\mathrm{r} 1}$ through the high-voltage power line $\mathrm{L}_{1}$ and the transformer $\mathrm{T}_{\mathrm{r} 2}$ feeds the load 2, at which the asynchronous generator ADFM WPP also operates.

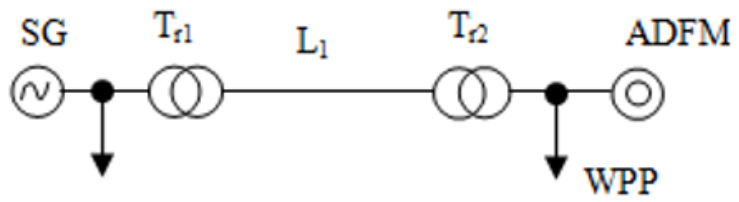

Load 1

Load 2
This scheme, of course, cannot be the only one $-\mathrm{T}_{\mathrm{r} 1}$ and $\mathrm{T}_{\mathrm{r} 2}$ may be absent, power takeoff may be in different places of the line $\mathrm{L}_{1}$, etc. But this scheme corresponds to the semantic content of the question under study, i.e., when the output power of the WPP's asynchronous generator changes depending on the wind speed, the power distribution on load 2 will change - when the output power of the WPP decreases, the main synchronous generator will be loaded, and when it increases-vise versa $[1,2,3,4,5]$.

The equivalent circuit of the power transmission scheme, presented in Fig.1, is shown in Fig. 2.

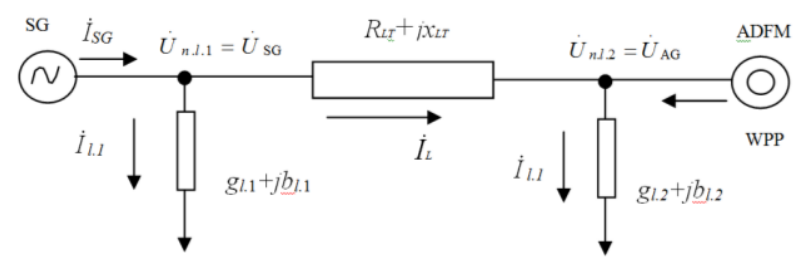

Fig. 2. Power Transmission Equivalent Schemes.

Here $\dot{I}_{l 1}, \dot{I}_{l 2}-$ currents of load 1 and load 2 in complex form, $\dot{I}_{L}-$ line current; $\left(g_{n 1}+j b_{n 1}\right)$ and $\left(g_{l 2}+j b_{l 2}\right)-$ components of the active $g_{l 1}, g_{l 2}$ and reactive $b_{l 1}, b_{l 2}$ conductivities of first and second loads respectively; $\left(r_{L \mathrm{~T}}+j x_{L \mathrm{~T}}\right)$ - concurrent resistances (active $r_{L \mathrm{~T}}$ and reactive $x_{L \mathrm{~T}}$ ) of power transmission line $\mathrm{L}_{1}$ and

Fig. 1. Equivalent Schemes. 
transformers $\mathrm{T}_{\mathrm{r} 1}$ and $\mathrm{T}_{\mathrm{r} 2} ; \quad U_{\text {l.n.1 }}=U_{\mathrm{SG}}, \quad U_{\text {l.n.2 }}=U_{\mathrm{AG}}-$ voltages of load nodes $\dot{U}_{l . n .1}$ and $\dot{U}_{l . n .2}$, which are equal to generator voltages of synchronous and asynchronous machines, $\dot{I}_{\mathrm{SG}}, \dot{I}_{\mathrm{AG}}-$ currents of synchronous and asynchronous generators in complex form.

Direction of 1 node is determined from the relationship:

$$
\dot{U}_{l . n .1}-\dot{U}_{\mathrm{SG}}=U_{l . n .2}^{\cdot}+\dot{I}_{L}\left(r_{L \mathrm{~T}}+j x_{L \mathrm{~T}}\right)
$$

Currents in nodes 1 and 2 are respectively as follows:

$$
\left.\begin{array}{l}
\dot{I}_{l 1}=\dot{U}_{l . n .1}\left(g_{l 1}+j b_{l 1}\right)=\dot{U}_{\mathrm{SG}}\left(g_{n 1}+j b_{l 1}\right) \\
\dot{I}_{l 1}=\dot{U}_{l . n .2}\left(g_{H 2}+j b_{l 2}\right)=\dot{U}_{\mathrm{AG}}\left(g_{l 2}+j b_{l 2}\right)
\end{array}\right\}
$$

Current balance:

$$
\left.\begin{array}{lll}
\dot{I}_{\mathrm{SG}}=\dot{I}_{L}+\dot{I}_{l 1} & \text { or } & \dot{I}_{L}=\dot{I}_{\mathrm{SG}}-\dot{I}_{l 1} \\
\dot{I}_{\mathrm{AG}}=\dot{I}_{l 2}-\dot{I}_{L} & & \dot{I}_{L}=\dot{I}_{l 2}-\dot{I}_{\mathrm{AG}}
\end{array}\right\}
$$

taking into account the last expression, the voltages of synchronous and asynchronous generators will be written as:

$$
\left.\begin{array}{l}
\dot{U}_{\mathrm{SG}}=\dot{U}_{\mathrm{AG}}+\left(\dot{I}_{\mathrm{SG}}-\dot{I}_{l 1}\right) \cdot\left(r_{L \mathrm{~T}}+j x_{\mathrm{LT}}\right) \\
\dot{U}_{\mathrm{AG}}=\dot{U}_{\mathrm{SG}}-\left(\dot{I}_{l 2}-\dot{I}_{\mathrm{AG}}\right) \cdot\left(r_{L \mathrm{~T}}+j x_{L \mathrm{~T}}\right)
\end{array}\right\}
$$

Then, substituting the load current values from equations (2) into the expression (3), we get:

$$
\left.\begin{array}{l}
\dot{U}_{\mathrm{SG}}=\dot{U}_{\mathrm{AG}}+\dot{I}_{\mathrm{SG}}\left(r_{L \mathrm{~T}}+j x_{L \mathrm{~T}}\right)-\dot{U}_{\mathrm{SG}}\left(g_{l 1}+j b_{l 1}\right) \cdot\left(r_{L \mathrm{~T}}+j x_{L \mathrm{~T}}\right) \\
\dot{U}_{\mathrm{AG}}=\dot{U}_{\mathrm{SG}}+\dot{I}_{\mathrm{AG}}\left(r_{L \mathrm{~T}}+j x_{L \mathrm{~T}}\right)-\dot{U}_{\mathrm{AG}}\left(g_{l 2}+j b_{l 2}\right) \cdot\left(r_{L \mathrm{~T}}+j x_{L \mathrm{~T}}\right)
\end{array}\right\}
$$

Grouping members, we obtain:

$$
\left.\begin{array}{l}
\dot{U}_{\mathrm{SG}}\left[1+\left(g_{l 1}+j b_{l 1}\right) \cdot\left(r_{L \mathrm{~T}}+j x_{L \mathrm{~T}}\right)\right]=\dot{U}_{\mathrm{AG}}+\dot{I}_{\mathrm{SG}}\left(r_{L \mathrm{~T}}+j x_{L \mathrm{~T}}\right) \\
\dot{U}_{\mathrm{AG}}\left[1+\left(g_{l 2}+j b_{l 2}\right) \cdot\left(r_{L \mathrm{~T}}+j x_{L \mathrm{~T}}\right)\right]=\dot{U}_{\mathrm{SG}}+\dot{I}_{\mathrm{AG}}\left(r_{L \mathrm{~T}}+j x_{L \mathrm{~T}}\right)
\end{array}\right\}
$$

At last, the last equations can be written in the following form:

$$
\left.\begin{array}{l}
\dot{U}_{\mathrm{SG}}=\dot{K}_{s} \cdot \dot{U}_{\mathrm{AG}}+\dot{K}_{L s} \dot{I}_{\mathrm{SG}} \\
\dot{U}_{\mathrm{AG}}=\dot{K}_{a} \cdot \dot{U}_{\mathrm{SG}}+\dot{K}_{\mathrm{La}} \cdot \dot{I}_{\mathrm{AG}}
\end{array}\right\}
$$

where $\dot{K}_{s}=\frac{1}{1+\left(g_{l 1}+j b_{l 1}\right) \cdot\left(r_{L \mathrm{~T}}+j x_{L \mathrm{~T}}\right)}$;

$$
\begin{aligned}
& \dot{K}_{L s}=\frac{\left(r_{L \mathrm{~T}}+j x_{L \mathrm{~T}}\right)}{1+\left(g_{l 1}+j b_{l 1}\right) \cdot\left(r_{L \mathrm{~T}}+j x_{L \mathrm{~T}}\right)} ; \\
& \dot{K}_{a}=\frac{1}{1+\left(g_{l 2}+j b_{l 2}\right) \cdot\left(r_{L \mathrm{~T}}+j x_{L \mathrm{~T}}\right)} ; \\
& \dot{K}_{L a}=\frac{\left(r_{L \mathrm{~T}}+j x_{L \mathrm{~T}}\right)}{1+\left(g_{l 2}+j b_{l 2}\right) \cdot\left(r_{L \mathrm{~T}}+j x_{L \mathrm{~T}}\right)} .
\end{aligned}
$$

Solving together the equations of the system (7), we get:

$$
\left.\begin{array}{c}
\dot{U}_{\mathrm{SG}}=\frac{\dot{K}_{s} \cdot \dot{K}_{L a}}{1-\dot{K}_{s} \cdot \dot{K}_{a}} \dot{I}_{\mathrm{AG}}+\frac{\dot{K}_{L s}}{1-\dot{K}_{s} \cdot \dot{K}_{a}} \dot{I}_{\mathrm{SG}} \\
\dot{U}_{\mathrm{AG}}=\frac{\dot{K}_{a} \cdot \dot{K}_{L s}}{1-\dot{K}_{s} \cdot \dot{K}_{a}} \dot{I}_{\mathrm{SG}}+\frac{\dot{K}_{L a}}{1-\dot{K}_{s} \cdot \dot{K}_{a}} \dot{I}_{\mathrm{AG}}
\end{array}\right\}
$$

For simplicity of further transformations these equations can be represented as:

$$
\left.\begin{array}{l}
\dot{U}_{\mathrm{SG}}=\dot{A} \cdot \dot{I}_{\mathrm{AG}}+\dot{B} \cdot \dot{I}_{\mathrm{SG}} \\
\dot{U}_{\mathrm{AG}}=\dot{C} \cdot \dot{I}_{\mathrm{SG}}+\dot{D} \cdot \dot{I}_{\mathrm{AG}}
\end{array}\right\}
$$

where $\dot{A}=\frac{\dot{K}_{s} \cdot \dot{K}_{L a}}{1-\dot{K}_{s} \cdot \dot{K}_{a}} ; \dot{B}=\frac{\dot{K}_{L s}}{1-\dot{K}_{s} \cdot \dot{K}_{a}} ; \dot{C}=\frac{\dot{K}_{a} \cdot \dot{K}_{L s}}{1-\dot{K}_{s} \cdot \dot{K}_{a}} ;$

$$
\dot{D}=\frac{\dot{K}_{L a}}{1-\dot{K}_{s} \cdot \dot{K}_{a}} \text {. }
$$

Now we write down the equations of the external network in the axes $d, q$, rotating at the speed of the synchronous machine rotor $[0,0]$. At the same time we superpose the $\mathrm{q}$ axis with the real numbers axis, and the $d$ axis with the imaginary numbers axis. Then the voltages and currents of generators can be written in the following form:

$$
\left.\begin{array}{l}
\dot{U}_{\mathrm{SG}}=U_{\mathrm{SG} q}+j U_{\mathrm{SG} d} \\
\dot{U}_{\mathrm{AG}}=U_{\mathrm{AG} q}+j U_{\mathrm{AG} d} \\
\dot{I}_{\mathrm{SG}}=i_{\mathrm{SG} q}+j i_{\mathrm{SG} d} \\
\dot{I}_{\mathrm{AG}}=U_{\mathrm{AG} q}+j i_{\mathrm{AG} d}
\end{array}\right\}
$$

Exactly the same representation is made for the external axis parameters:

$$
\left.\begin{array}{ll}
\dot{A}=a_{q}+j a_{d} ; & \dot{C}=c_{q}+j c_{d} \\
\dot{B}=b_{q}+j b_{d} ; & \dot{D}=d_{q}+j d_{d}
\end{array}\right\}
$$

Substituting the values of the relationships (10) and (11) in equations (9), and making the corresponding multiplication, we obtain for the synchronous generator:

$$
\left.\begin{array}{l}
U_{\mathrm{SG} q}=a_{q} \cdot i_{\mathrm{AG} q}-a_{d} \cdot i_{\mathrm{AG} d}+b_{q} \cdot i_{\mathrm{SG} q}-b_{d} \cdot i_{\mathrm{SG} d} \\
U_{\mathrm{SG} d}=a_{q} \cdot i_{\mathrm{AG} d}+a_{d} \cdot i_{\mathrm{AG} q}+b_{q} \cdot i_{\mathrm{SG} d}+b_{d} \cdot i_{\mathrm{SG} q}
\end{array}\right\}
$$

and for asynchronous generator:

$$
\left.\begin{array}{l}
U_{\mathrm{AG} q}=c_{q} \cdot i_{\mathrm{SG} q}-c_{d} \cdot i_{\mathrm{SG} d}+d_{q} \cdot i_{\mathrm{AG} q}-d_{d} \cdot i_{\mathrm{AG} d} \\
U_{\mathrm{AG} d}=c_{q} \cdot i_{\mathrm{SG} d}+c_{d} \cdot i_{\mathrm{SG} q}+d_{q} \cdot i_{\mathrm{AG} d}+d_{d} \cdot i_{\mathrm{AG} q}
\end{array}\right\}
$$

In equations (12) and (13): $U_{\mathrm{SG} q}, U_{\mathrm{SG} d}, i_{\mathrm{SG} q}, i_{\mathrm{SG} d}-$ components of the synchronous generator voltages and currents along the axes $d, q$, rotating at the speed of the synchronous generator rotor; $U_{\mathrm{AG} q}, U_{\mathrm{AG} d}, i_{\mathrm{AG} q}, i_{\mathrm{AG} d}-$ components of voltages and currents of the WPP's asynchronous generator, performed on the basis of double-fed machine, written in axes $d, q$ rotating also at the speed of the synchronous generator rotor. The 
transition from the variables $i_{\mathrm{AG} q}^{0}$ and $i_{\mathrm{AG} d}^{0}$, written in the axes $d_{a}$ and $q_{a}$, rotating at the speed of the asynchronous generator rotor, to the variables $i_{\mathrm{AG} q}$ and $i_{\mathrm{AG} d}$, determined by the following relationships.

$$
\left.\begin{array}{l}
i_{\mathrm{AG} q}=-i_{\mathrm{AG} d}^{0} \sin \theta_{s a}+i_{\mathrm{AG} q}^{0} \cos \theta_{s a} \\
i_{\mathrm{AG} d}=i_{\mathrm{AG} d}^{0} \cos \theta_{s a}+i_{\mathrm{AG} q}^{0} \sin \theta_{s a}
\end{array}\right\}
$$

where $\theta_{s a}-$ the transverse axis angle of the WPP's asynchronous generator relative to the transverse axis of the synchronous generator, i.e. $\theta_{s a}=\theta_{s}-\theta_{a}$.

Accordingly, the components of the asynchronous generator voltage in the axes rotating at the speed of the WPP's asynchronous generator rotor can be represented as follows:

$$
\left.\begin{array}{l}
U_{\mathrm{AG} q}^{0}=U_{\mathrm{AG} d} \sin \theta_{s a}+U_{\mathrm{AG} q} \cos \theta_{s a} \\
U_{\mathrm{AG} d}^{0}=U_{\mathrm{AG} d} \cos \theta_{s a}-U_{\mathrm{AG} q} \sin \theta_{s a}
\end{array}\right\}
$$

Thus, taking into account the above, the equations of the synchronous generator in the axes $\mathrm{d}, \mathrm{q}$, rotating at the speed of its rotor $[8,9]$, are represented in the form:

$$
\begin{aligned}
& p \Psi_{d s s}=U_{\mathrm{SG} d}-\omega_{r s} \cdot \Psi_{q s s}-r_{s s} \cdot i_{r s d} \\
& p \Psi_{q s c}=U_{\mathrm{SG} q}+\omega_{r s} \cdot \Psi_{d s s}-r_{s s} \cdot i_{r s q} \\
& p \Psi_{d r s}=-\frac{r_{d r s}}{x_{d r s}} \cdot \Psi_{d r s}+\frac{r_{d r s} \cdot x_{d d s}}{x_{d r s}} \cdot i_{\mathrm{SG} d}+\frac{r_{d r s} \cdot x_{a d s}}{x_{d r s}} \cdot i_{d f s} \\
& p \Psi_{q r s}=-\frac{r_{q r s}}{x_{q r s}} \cdot \Psi_{q r s}+\frac{r_{q r s} \cdot x_{a q s}}{x_{q r s}} \cdot i_{\mathrm{SG} q} \\
& p \Psi_{d f s}=\frac{r_{d f s}}{x_{a d s}} \cdot U_{d f s}-r_{d f s} \cdot i_{d f s} \\
& p \omega_{r s}=\frac{1}{T_{j}} \cdot m-\frac{1}{T_{j}} \cdot m_{e m s} \\
& m_{e m s}=\Psi_{d s s} \cdot i_{\mathrm{SG} q}-\Psi_{q s s} \cdot i_{\mathrm{SG} d} \\
& i_{\mathrm{SG} d}=\frac{x_{q r s}}{\Delta d_{s}} \cdot \Psi_{d s s}-\frac{\Delta d_{1 s}}{\Delta d_{s}} \cdot i_{d f s}-\frac{x_{a d s}}{\Delta d_{s}} \cdot \Psi_{d r s} \\
& i_{\mathrm{SG} q}=\frac{x_{q r s}}{\Delta q_{s}} \cdot \Psi_{q s s}-\frac{x_{a q s}}{\Delta q_{s}} \cdot \Psi_{q r s} \\
& i_{d f s}=\frac{x_{d r s}}{\Delta d_{2 s}} \cdot \Psi_{d f s}-\frac{\Delta d_{1 s}}{\Delta d_{2 s}} \cdot i_{S G d}-\frac{x_{a d s}}{\Delta d_{2 s}} \cdot \Psi_{d r s} \\
& p \theta=\omega_{r s}+1
\end{aligned}
$$

where $\quad \Delta d_{s}=x_{d s s} \cdot x_{d r s}-x_{a d s}^{2} ; \quad \Delta q_{s}=x_{q s s} \cdot x_{q r s}-x_{a q s}^{2} ;$ $\Delta d_{1 s}=x_{d r s} \cdot x_{a d s}-x_{a d s}^{2} ; \Delta d_{2 s}=x_{d r s} \cdot x_{d f s}-x_{a d s}^{2}$.

The voltages $U_{\mathrm{SG} d}, U_{\mathrm{SG} q}$ are determined based on the equations (13), in which the currents $i_{\mathrm{AG} d}, i_{\mathrm{AG} q}$ are determined based on the expression (14).

The asynchronous generator equations - double-fed machines in axes $d_{a}, q_{a}$ rotating at the speed of the asynchronous generator rotor $[0,0]$ can be represented as:

$$
\begin{aligned}
& p \Psi_{d s a}=U_{\mathrm{AGd}}^{0}+\Psi_{q s a} \cdot\left(1-s_{a}\right)-r_{s a} \cdot i_{\mathrm{AGd}}^{0} \\
& p \Psi_{q s a}=U_{\mathrm{arq} q}^{0}-\Psi_{d s a} \cdot\left(1-s_{a}\right)-r_{s a} \cdot i_{\mathrm{AGq}}^{0} \\
& p \Psi_{d r a}=-U_{\mathrm{AG}}^{0} \cdot k_{u r} \cdot \sin \left(k_{f r} \cdot \tau\right)-r_{r a} \cdot i_{d r a} \\
& p \Psi_{q r a}=U_{\mathrm{AG}}^{0} \cdot k_{u r} \cdot \cos \left(k_{f r} \cdot \tau\right)-r_{r a} \cdot i_{q r a} \\
& p s_{a}=-\frac{1}{T_{j}} \cdot m-\frac{1}{T_{j}} \cdot m_{e m a} \\
& m_{e m a}=\Psi_{d s a} \cdot i_{\mathrm{AG} q}^{0}-\Psi_{q s a} \cdot i_{\mathrm{AG} d}^{0} \\
& i_{\mathrm{AGd}}^{0}=k_{s a} \cdot \Psi_{d s a}-k_{m} \cdot \Psi_{d r a} \\
& i_{\mathrm{AG} q}^{0}=k_{s a} \cdot \Psi_{q s a}-k_{m} \cdot \Psi_{q r a} \\
& i_{d r a}=k_{r a} \cdot \Psi_{d r a}-k_{m} \cdot \Psi_{d s a} \\
& i_{q r a}=k_{r a} \cdot \Psi_{q r a}-k_{m} \cdot \Psi_{q s a} \\
& p \theta_{a}=s_{a} \quad \text { and } \quad \theta_{s a}=\theta_{s}-\theta_{a}
\end{aligned}
$$

where

$$
k_{s a}=\frac{x_{r a}}{x_{r a} \cdot x_{s a}-x_{m a}^{2}} ; \quad k_{r a}=\frac{x_{s a}}{x_{r a} \cdot x_{s a}-x_{m a}^{2}} ;
$$

$$
k_{m}=\frac{x_{m a}}{x_{r a} \cdot x_{s a}-x_{m a}^{2}} ; U_{\mathrm{AG}}^{0}=\sqrt{\left(U_{\mathrm{AG} d}^{0}\right)^{2}+\left(U_{\mathrm{AG} q}^{0}\right)^{2}} .
$$

In the system of equations (17), the stator voltage components $U_{\mathrm{AG} d}$ and $U_{\mathrm{AG} q}$ are determined from the equations (15).

\section{Results and discussion}

To study the adequacy of the presented equations (16) and (17), the parameters of the elements and the coefficients of the equations were calculated.

a) As a synchronous generator, a generator of the FWTG type (forced water turbo-generator) -100-2 [12] with parameters: $P_{l}=100 \mathrm{MW} ; U_{L n}=10.5 \mathrm{kV} ; \cos \varphi=0.85$ $(\sin \varphi=0.527) ; \eta=0.983$.

As a result of the calculations, the generator parameters are determined in relative units: $x_{d}=1,1$; $x_{q}=0,73 ; x_{\sigma}=0,16 ; x_{a d}=0,94 ; x_{a q}=0,57 ; x_{f}=1,26$; $x_{r d}=1,16 ; \quad x_{r q}=0,735 ; \quad r_{s}=0,008 ; \quad r_{r d}=0,0094$; $r_{r q}=0,00765 ; T_{j}=7 \mathrm{~s} . ; \frac{1}{T_{j}}=0,00045[\mathrm{rad}$.$] .$

b) 125 MVA $110 \mathrm{kV}$ type transformer with power $S_{n}=125$ MVA is accepted as I step-up transformer, voltage $110 / 10.5 \mathrm{kV}$. No-load losses $\Delta P_{n . l}=105 \mathrm{~kW}$; short-circuit losses $\Delta P_{\text {s.c. }}=400 \mathrm{~kW}$; short-circuit voltage in $\% U_{n . l .}=11 \%$; no-load current in $\% I_{n . l}=0.55 \%$; $\cos \varphi_{1}=0.8[0]$.

c)AS-120 grade conductor with parameters $r_{L}^{*}=0.27 \mathrm{Ohm} / \mathrm{km}, x_{L}^{*}=0.379 \mathrm{Ohm} / \mathrm{km}$ is accepted for $110 \mathrm{kV}$ OHL, connecting node 1 with node 2 [0].

Accepting the total length of the line $30 \mathrm{~km}$, we get: $r_{L}=0.27 \cdot 30=8.1 \mathrm{Ohm} ; x_{L}=0.379 \cdot 30=11.37 \mathrm{Ohm}$.

d) 25 MVA $110 \mathrm{kV}$ type transformer with power $S_{l}=25 \mathrm{MVA}$ is accepted as step-down transformer Tr2, $U_{h . v .}=100 \mathrm{kV}$, no-load losses $\Delta P_{n . l .}=25 \mathrm{~kW}$, short-circuit losses $\Delta P_{\text {s.c. }}=120 \mathrm{~kW}$, short-circuit voltage in $\%$ $U_{\text {s.c. }}=10.5 \%$, no-load current in $\% I_{n . l .}=0.65 \%$. 
Thus, total resistance of line and transformers

$r_{L \mathrm{~T}}=0.074+0.0135+0.0018=0.089 \mathrm{Ohm}$

$x_{L \mathrm{~T}}=0.1+0.369+0.077=0.546 \mathrm{Ohm}$

These resistances in relative units given in $Z_{b a z}$ of the synchronous generator

$$
\begin{aligned}
& r_{L \mathrm{~T}}=\frac{0.089}{0.92} \approx 0.097 \\
& x_{L \mathrm{~T}}=\frac{0.546}{0.92} \approx 0.59 .
\end{aligned}
$$

e) Load parameters for steady-state mode.

The total load of the synchronous generator as indicated $P_{l . s .}=100 \mathrm{MW} ; Q_{l . s .}=60 \mathrm{MVAr}$.

Let's assume the active and reactive loads of the $1 \mathrm{st}$ node equal to: $P_{l .1}=60 \mathrm{MW} ; Q_{l .1}=30 \mathrm{MVAr}$; and of the 2nd node, $P_{l .2}=40 \mathrm{MW}, Q_{l .2}=20 \mathrm{MVAr}$ respectively.

f) Generator made on the basis of double-fed machine, which, of course, is an asynchronous machine with a phase rotor, is accepted as WPP's asynchronous generator $[0,0]$. The power of one generator is assumed to be equal to $P_{l}=20 \mathrm{MW}$, which is equivalent to 10 WPPs, each of which has the power of $2 \mathrm{MW}$.

Thus, parameters of equivalent generator of WPS in relative units at $Z_{b a z}=4.5 \mathrm{Ohm}$ are equal to:

$$
\begin{aligned}
& r_{1}^{*}=\frac{0.05}{4.5}=0.011 ; r_{2}^{* *}=\frac{0.058}{4.5}=0.0128 ; x_{\sigma 1}^{*}=\frac{0.45}{4.5}=0.1 ; \\
& x_{\sigma 2}^{*}=\frac{0.52}{4.5}=0.115 ; x_{m}^{*}=\frac{13.66}{4.5}=3.035 ; \\
& x_{1}^{*}=x_{\sigma 1}^{*}+x_{m}^{*}=3.035+0.1=3.135 ; x_{2}^{*}=3.035+0.115=3.15 .
\end{aligned}
$$

$g$ ) Calculation of coefficients of external network equations:

$$
\begin{gathered}
\quad a_{q}=-0,124 ; \quad b_{q}=0,304 ; \quad c_{q}=-0,124 ; \quad d_{q}=0,159 ; \\
a_{d}=-0,043 ; b_{d}=-0,172 ; c_{d}=1,69 ; d_{d}=-0,13 .
\end{gathered}
$$

\section{Conclusions}

Dynamic characteristics have represented the discrete change process of the voltage and part of the current of the rotor, at which the speed of the WPP shaft changes within $\pm 22 \%$.

Model of operation of WPP with a double-fed machine in parallel with a synchronous generator is proposed. At the same time, the WPP power is assumed to be about $20 \%$ of the synchronous generator power.

\section{References}

[1] S.P. Gawande, K.B. Porate, Review of Parallel Operation of Synchronous Generator and Induction Generator for Stability, Conference: Proceedings of the Second International Conference on Emerging Trends in Engineering \& Technology, ICETET 2009, Nagpur, Maharashtra, India, 16-18 December (2009). DOI: 10.1109/ICETET.2009.193 (2009)

[2] J. Liu, M. Yushi, T. Ise, J. Yoshizawa and K. Watanabe, Parallel operation of a synchronous generator and a virtual synchronous generator under unbalanced loading condition in microgrids, 2016 IEEE $8^{\text {th }}$ International Power Electronics and Motion Control Conference (IPEMC-ECCE Asia), Hefei, 3741-3748. DOI: 10.1109/IPEMC.2016.7512894 (2016)

[3] V.Z. Silva, Â.J. Junqueira Rezek, R. Di, L.Corrêa, Analysis of synchronous and induction generators in parallel operation mode in an isolated electric system, IEEE 8th International Symposium on Power Electronics for Distributed Generation Systems DOI: 10.1109/PEDG40096.2017 (2017)

[4] D. Harikrishna, N.V. Srikanth, Dynamic Stability Enhancement of Power Systems Using NeuralNetwork Controlled Static-Compensator, TELKOM-NIKA 10, 1, 9-16 (2012)

[5] I. Tamrakar, L.B. Shilpakar, B.G. Fernandes, R. Nilsen, Voltage and frequency control of parallel operated synchronous generator and induction generator with STATCOM in micro hydro scheme 1, 5, 743-750. DOI: 10.1049/ietgtd:20060385 (2007)

[6] L. Congwei, W. Haiqing, S. Xudong, L. Fahai, Research of stability of double fed induction motor vector control system, Shenyang, China, Proc. of the Fifth International Conference on Electrical Machines and Systems 2, 1203-1206, 18-20 Aug. (2001)

[7] A.P. Sokolov, O.V. Gazizova, Yu.N. Kondrashova, Study of the transients with the loss of field of the synchronous generator in the industrial electric power station, Quality Management and Reliability of Technical Systems, IOP Conf. Series: Materials Science and Engineering 666, 1-7 (2019)

[8] R.I. Mustafaev, L.G. Hasanova, Modeling and study of the operating modes of synchronous generators of wind power plants with frequency control, Electricity, 7, 34-40 (2010)

[9] R.I. Mustafaev, L.G. Gasanova, An investigation of the dynamics of a wind power unit equipped with synchronous generators with permanent magnets, Russian Electrical Engineering 86, 6, 258-263 (2015)

[10] M. Heller, W. Schumacher, Stability analysis of doubly-fed induction machines in stator flux reference frame, Brussels, Belgium, Proc. of 7th European Conference on Power Electronics and Applications 2, 707-710, 8-10 Sept. (1997)

[11] H. Akagi, H Sato, Control and performance of a doubly-fed induction machine intended for a flywheel energy storage system, IEEE Transactions on Power Electronics 17, 1, 109116. DOI: https://doi.org/10.1109/63.988676 (2002)

[12] D.P. Kothari, I.J. Nagrath, Electric Machines (Tata McGraw-Hill Education, 834, 2004)

[13] I.A. Baumstein, S.A. Bazhenova, High Voltage Electrical Reference (3rd ed., Revised. and add., Moscow: Energoatomizdat, 768, 1989) 
[14] R.I. Mustafayev, L.H. Hasanov, M.M. Musayev, E.M. Mamedov, X.İ. Nabiyev, Modeling and analysis of operating regimes of hydraulic units of small hydropower plant with double fed machine, Electromechanics, 6, 59-65 (2015)

[15] A. Dittrich, A. Stoev, Grid voltage fault proof doubly-fed induction generator system, Proc. Power Electronics and Applications (EPE), Toulouse, France (2003)

[16] J.W. Park, K.W. Lee, D.W. Kim, et al., Control Method of a Doubly-Fed Induction Generator with a Grid Synchronization against Parameter Variation and Encoder Position, Industry Applications Conference, 42nd IAS Annual Meeting, Conference Record of 2007 IEEE, New Orleans, LA, 931-935 (2007) 